\title{
Reply to does the survival between elderly and very elderly patients with different malignant primary bone tumours have no difference?
}

\author{
Lechler Philipp
}

Received: 30 October 2014 / Accepted: 30 October 2014 / Published online: 4 December 2014

(C) SICOT aisbl 2014

Dear Editor,

We thank Dr. Li and colleagues for their interest and critical analysis of the article "Surgical outcome of malignant primary bone tumours in elderly and very elderly patients" [1] which was published in the October edition of your esteemed journal.

Three key issues were raised:

Re 1:

The authors of the letter to the editor emphasise the heterogeneity of primary bone tumours in the presented cohort and highlight the role of histological grade for patient survival. While we agree with Li et al. on the importance of histopathological tumour type and grade, the primary research question of our study was to examine the age-dependent clinico-pathological and treatment characteristics and surgical outcome of elderly patients with primary malignant bone tumours. For reasons of transparency, Tables 1, 2 and 3 give comprehensive information on patient details including tumour grade. Due to the relatively small number of distinct tumour entities in each age group, we believe a further subgroup analysis is not appropriate. However, we support the suggestion of $\mathrm{Li}$ et al. and recommend future studies specifically designed to analyse the role of tumour grade and entity on the outcome in elderly patients with primary bone tumours.

Re 2:

All statistical methods applied were described in the section "statistical analysis". The tabular data presented are descriptive only; a direct statistical comparison regarding the clinico-pathological data of the subgroups was not performed. The authors are convinced that the application of further explorative statistical methods would not have improved the interpretability of the data presented; conclusions would have been severely limited by the multitude of the factors analysed and the relatively small number of cases.

Re 3:

$\mathrm{Li}$ and colleagues mention the calculation of effective sample sizes for clinical studies. While sample size calculations are a necessity during the planning of prospective clinical trials, retrospective designs are usually restricted by the available number of cases, in particular in studies on rare diseases. As mentioned earlier (re 1), we agree that the sample sizes of the different tumour types do not suffice for statistical analysis, which was consequently not performed.

Finally, the study highlights the importance of further research on the treatment and outcome in the population of elderly patients with malignant primary bone tumours.

\section{Reference}

1. Cosker T, Lechler P, Gulati A, Whitwell D, Giele H, Trent S, Athanasou N, Gibbons C (2014) Surgical outcome of malignant primary bone tumours in elderly and very elderly patients. Int Orthop 38(10):2149-2154. doi:10.1007/s00264-014-2400-4

\author{
L. Philipp $(\bowtie)$ \\ Department of Trauma, Hand and Reconstructive Surgery, \\ University of Giessen and Marburg, Marburg, Germany \\ e-mail: lechler@med.uni-marburg.d \\ L. Philipp \\ Oxford Sarcoma Service, Nuffield Orthopaedic Centre, Oxford, UK
}

